\title{
Cloud Cover Analysis from Satellite Imagery Using Spatial and Temporal Characteristics of the Data
}

\author{
Genevieve Seze AND Michel Desbois \\ LMD/CNRS, 91128 PALAISEAU CEDEX (France)
}

(Manuscript received 6 June 1985, in final form 29 October 1986)

ABSTRACT

\begin{abstract}
New developments of a cloud classification scheme based on histogram clustering by a statistical method are presented. Use of time series of geostationary satellite pictures as well as for construction of composite images representative of the surface properties and then for the identification of significative cloud classes is discussed. Spatial variances are introduced as additional parameters of the classification, with the aim to better separate clouds from the surface and the different kinds of more or less homogeneous cloud classes.
\end{abstract}

\section{Introduction}

During the last few years, research on cloud characterization from multispectral satellite imagery has been stimulated by the International Satellite Cloud Climatology Program (ISCCP). Several methods of cloud classification have been tested in a systematic algorithm intercomparison (Rossow et al., 1985).

All these methods consist of two steps. The first step, cloud detection, determines the percentage of cloud cover. The second step, analysis, determines the properties of the clouds found in the first step. These methods can be separated into two groups: 1) threshold methods where the cloud detection and analysis are performed on individual pixels using VIS and IR radiances and physical thresholdings; and 2) statistical methods where these two steps are not performed on each pixel, but globally on image segments, using the statistical properties of the VIS and IR radiances. (Rossow et al. 1986, Minnis and Harrison 1984, Desbois et al. 1982, Simmer et al. 1982, Phulpin et al. 1983). In most of the cases, statistical and threshold methods agree reasonably well. But all the methods encounter similar problems-e.g., dependence on surface properties, nonblack clouds with varying emissivities and partial coverage of the pixels. Although statistical methods hold more potential for resolving these problems than do the threshold methods, at the time of the ISCCP intercomparison, statistical methods were not sufficiently developed and were insufficiently tested to be applied operationally. Indeed, for the ISCCP operations, a threshold method has been chosen using auxiliary data as ground truth data and other satellite measurements. However, development of statistical methods must be pursued (Rossow et al. 1985) to extract the maximum information from the VIS and IR images, in the aim to reduce the need of auxiliary data and to have a better understanding of the cloud properties variability.

The statistical methods basically start from the spectral representation of one image segment or repartition function of the pixels of this image segment in the spectral space. This repartition function is called a bior multidimensional histogram depending on the number of spectral channels. Assuming that pixels of one cloud layer or surface have homogeneous (VISIR) spectral signatures, a surface or cloud type is represented by a high density peak in the histogram. Therefore, the statistical methods use clustering techniques to obtain histogram partitions into clusters centered on these peaks. Clusters are assumed to be representative of cloud or surface classes.

Intercomparisons have shown that the clusters found on the same sample were often method dependent, e.g., between the Dynamic Cluster method (Desbois et al. 1982) and the Asymmetric Gaussian method (Simmer et al. 1982). This is attributed to the imprecise definition of the clusters on the histograms which often show no distinct peak, but a mixture of many intermediate values. Suggestions have been proposed to improve the process of cluster definition for these statistical methods. These include

- choosing more representative samples of the cloud population for the classification, by using larger areas and/or time-cumulated samples (Desbois and Seze, 1984);

- using spatial properties of the image, e.g., local spatial variances, to separate the homogeneous regions from intermediate points, interpreted as partially covered fields of view by Coakley and Bretherton (1982).

The purpose of this paper is to introduce into a statistical classification scheme both improved time sampling and spatial variances and to test the results ob- 
tained against a simple threshold technique, and manual nephanalysis. The integration of spatial and temporal properties of the data in the classification is done by using the repartition functions of images time series in the spectral (radiances), and spatial (local variance of radiances) spaces. Time series are currently used to construct images taken as representative of clear sky conditions, e.g., in the threshold methods (Minnis and Harrison, 1984; Rossow et al., 1985). Here we have used time series to determine surface radiances and study their temporal stability and to obtain radiances representative of cloud classes.

In section 2 the stability of surface conditions in time is tested by using different compositing techniques. In section 3 the usefulness of time cumulative (VISIR) bidimensional histograms for separating major cloud types is investigated. In section 4 the information contained in both VIS and IR spatial variances is discussed. Application of a classification scheme which uses both time sampling and spatial variances is presented in section 5 . In section 6 , cloud/surface determinations resulting from this classification scheme are compared with results of a simple threshold method using the clear sky reference images described in section 2. Finally, in section 7, a comparison of cloud types derived from the method used here and manual nephanalysis made on the same images is presented.

\section{Reference surface images and compositing tech- niques}

For a given period, threshold methods, using time series of satellite images to build "clear sky" maps, as well as a classification scheme working on time cumulated histograms, require that surface properties stay relatively constant during this period; in addition, atmospheric conditions should remain relatively stable if a correction for atmospheric effects is not undertaken. Fulfillment of these conditions was tested over a particular time series, which is used throughout this paper: it is composed from Meteosat visible $(0.4-1.1 \mu)$ and infrared (10.5-12.5 $\mu$ ) images over western Europe for the period 22 July-10 August 1983 . Only one image is chosen for each day (1130 UTC-scanning over Europe 1150 UTC) in order to have the best solar illumination. Full resolution Meteosat images are used (resolution at subsatellite point of $5 \mathrm{Km}$ in IR image and $2.5 \mathrm{Km}$ in VIS image). A sampling of one pixel out of four is applied to the VIS image to match the IR resolution.

From a meteorological point of view, the beginning of this period was characterized by a dust event coming from North Africa and spreading over the Mediterranean sea, associated with very warm temperatures in Europe, especially during the last days of July. The later part of the period was characterized by many kinds of clouds.

To avoid the use of varying accuracy in absolute calibration factors and angular or atmospheric correc- tions, all the analyses presented here are done directly on Meteosat counts, instead of on temperature and albedo. According to Esoc's calibration reports and B3 data set (ISCCP), a correspondence is given between VIS (IR) count values and albedo (apparent temperature).

Before applying any processing to these data, we assessed the stability of observation conditions:

- the calibration factor of both radiometers of the satellite has to remain stable during the period;

- solar zenith angle should not change too much through the analysis area or throughout the time period, due to seasonal variation of the inclination of the sun.

The first condition is verified according to Esoc's calibration reports; for the second, orders of magnitude of the reflectivity corrections at $45^{\circ}$ latitude due to the change of solar zenith angle were estimated according to a $\cos \theta$ law:

- $5 \%$ for the same pixel, from the beginning to the end of the period;

- $1 \%$ for $1^{\circ}$ of latitude difference on the same image;

- $0.3 \%$ for $1^{\circ}$ of longitude on the same picture.

Taking into account the generally low surface albedo in the region studied $(20 \%)$ and the poor radiometric resolution of Meteosat VIS radiometer (of the order of $2 \%$ in albedo), corrections of the order of $5 \%$ represent at most $1 \%$ in albedo, and can then be neglected.

Then, the main effect to take into account is the correction with latitude. For this reason, appropriate areas restricted in latitude have to be chosen for statistical classifications. Two of these areas are shown in Fig. 1. However, the construction of composite images can be applied directly on the whole image, each pixel being processed separately.

Currently, the most frequently used composite images are minimum brightness images like the one presented on Fig. 1. The minimum VIS value of one pixel during the whole period is kept as representative of the "clear sky conditions" for this pixel. This is based on the assumption that every time a cloud is present the brightness is enhanced. This image presents a good homogeneity except for some noise introduced by small dark features. Most of them can be attributed to the shadows of high clouds (Fig. 2). Reflectivity underestimation can also be due to presence of dust over bright surface.

We have also constructed a relative minimum brightness composite image for the same period. This composite, for each pixel location, is made up of the visible value corresponding to the maximum IR temperature day during the period. If the maximum temperature is reached more than once, the lowest corresponding VIS value is used. This is based on the assumption that no cloud is warmer than the warmer underlying surface during the whole period, and that the warmer value found is related to the clearer at- 


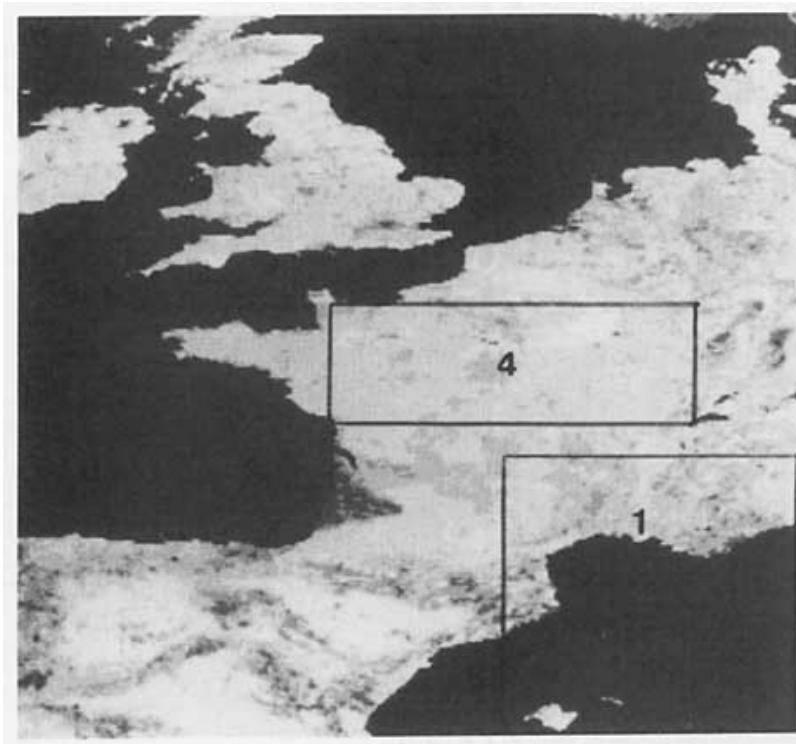

Fig. 1. Meteosat minimum brightness image at 1150 UTC for the period 22 July-10 August 1983, showing the region studied. Rectangular areas 1 and 4 represent the two areas specially described in this paper.

mosphere. The maximum temperature composite image is shown in Fig. 3 and the relative minimum brightness composite image is shown in Fig. 4. The relative minimum composite image looks very similar to the absolute minimum VIS image of Fig. 1, but is clearly noisier over sea. The differences between these two composites images are more obvious in the difference image (Fig. 5). In this image, increasing light tones indicate increasing differences between the absolute

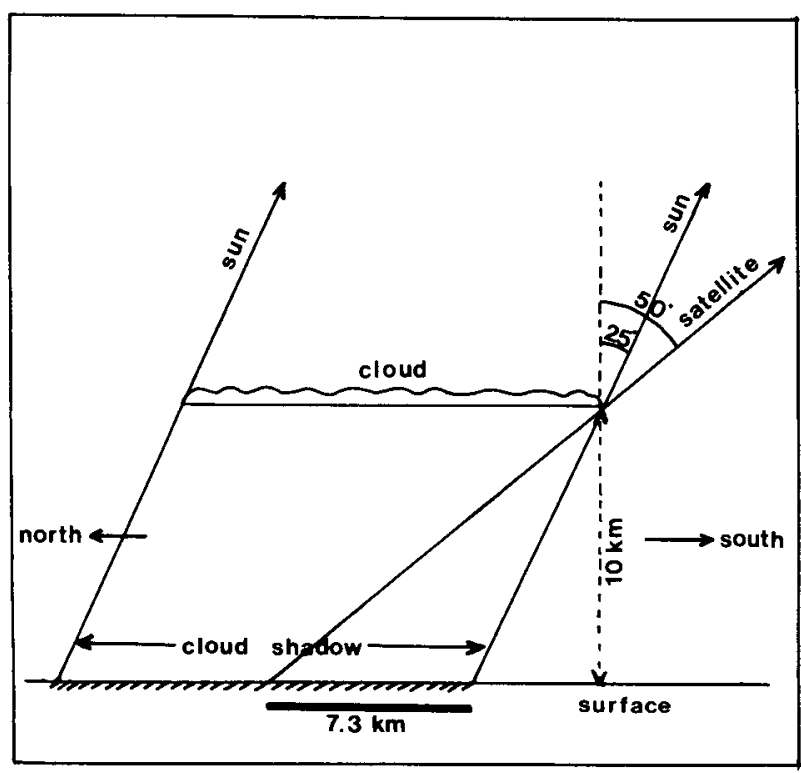

FIG. 2. Scheme of the geometry of illumination and observation for the studied images, showing how high cloud shadows can be seen from the satellite.

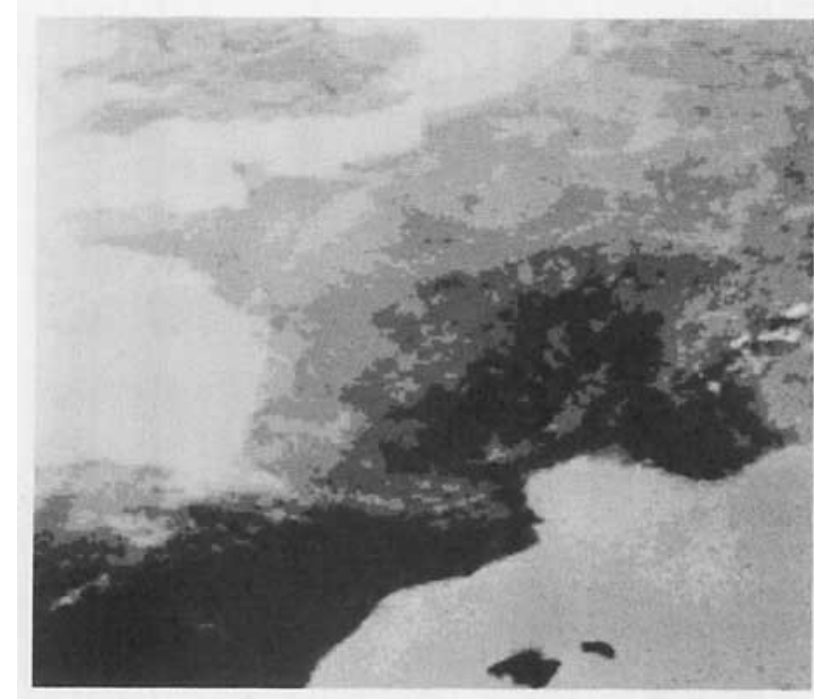

FIG. 3. Maximum IR radiance image at 1150 UTC for the period 22 July-10 August 1983.

minimum and the relative minimum VIS values. The linear features are a coastline artifact.

A careful examination of the days when the minimum VIS and the maximum IR occur in two different regions allows better understanding of these differences. The left panels of Fig. 6a and b, show for each daily image, the percentage of pixels reaching their minimum brightness and percentage of pixels reaching their maximum temperature for these two regions. These percentages have been computed with a radiometric resolution corresponding approximately to $2 \%$ in albedo and $1{ }^{\circ} \mathrm{C}$ in apparent temperature (for surface temperature). It appears that VIS and IR counts of one pixel do not always reach their extrema values at the

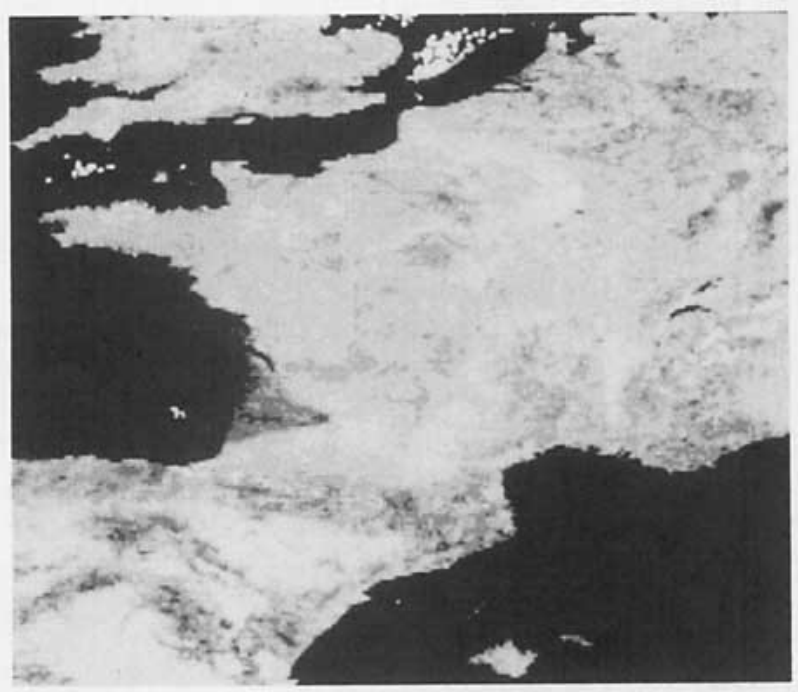

FIG. 4. Relative minimum brightness composite image composed of the pixels corresponding to maximum IR radiance (shown in Fig. 3). 


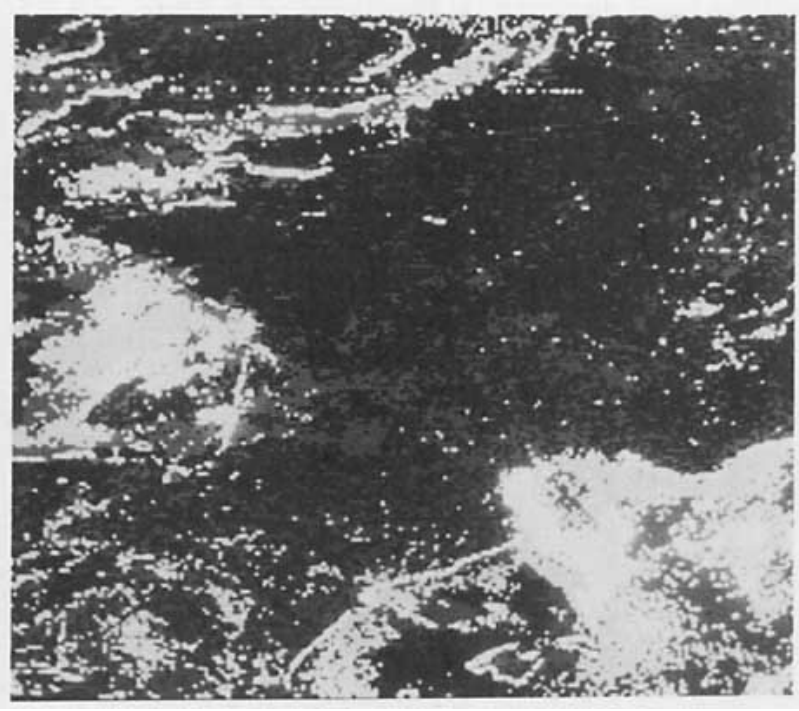

FIG. 5. Difference image between minimum VIS image (shown in Fig. 1) and composite VIS image (shown in Fig. 4).

same time; this is clear on Fig. 6a representing the mostly maritime region in the northwestern Mediterranean Sea. Also, it can be seen that minimum VIS values are reached several times, whereas a IR maximum value is reached mostly on one particular day, 30 July. In the case of Fig. 6b, which is taken over land in region 4, most of the IR maxima and VIS minima are reached on the same day, $30 \mathrm{July}$; for the IR, this is the case for $87 \%$ of the pixels, among which $77 \%$ reach the maximum only for this day. For the VIS, $85 \%$ of the pixels reach their minimum value on 30 July, among which $10 \%$ reach their minimum only $10 \%$ for this day. This indicates that on some days of the period some pixels without any detectable clouds from their VIS radiance can have an IR radiance smaller.

To look at the stability of the different kinds of composite images, we constructed minimum VIS and maximum IR composite images from a series of 19 days in which the particular case of 30 July was removed. The new minimum VIS image is very similar to the previous one (Fig. 1) and for a given day, the number of pixels reaching their minimum value does not change much. The new maximum IR image presents an average apparent temperature $2.5^{\circ} \mathrm{C}$ colder than the previous one (Fig. 3). For individual days, the number of pixels reaching their maximum IR values increases. These pixels appear each day in specific areas; the resulting maximum image is composed of a mosaic of these areas. This mosaic appears homogeneous, without discontinuities between the different areas.

Even on the 19 day series, minimum VIS values are reached much more often ( 3 times on the average) than IR maxima ( 1.4 times on the average). That cannot be attributed to the problem of radiometric resolution which is better in IR $\left(1^{\circ} \mathrm{C}\right.$ in apparent temperature) than in VIS ( $2 \%$ in albedo). Indeed, we have degraded the IR radiometric resolution from 128 levels (temperature resolution of $1^{\circ} \mathrm{C}$ ) to 64 levels (temperature resolution of $2^{\circ} \mathrm{C}$ ), and then to 32 levels $\left(4^{\circ} \mathrm{C}\right)$. Even in this case, IR maxima are reached less than two times, on average, during the whole series. This can be due to the following causes:

1) Natural variability of surface temperature. The meteorological air temperature during this period at $1200 \mathrm{UTL}$ did vary from about $20^{\circ} \mathrm{C}$ to more than $30^{\circ} \mathrm{C}$ on some days like on $30 \mathrm{July}$. It appears, by comparison with the satellite images, that the lowest meteorological temperatures are associated with cloud occurrence, and the spatial homogeneity of the IR maximum image seems to demonstrate that the variability of surface temperature under clear air conditions is smaller.

2) Presence of thin high clouds not detectable in the VIS channel. Indeed, among the points reaching their minimum value in the visible, more than $13 \%$ correspond to apparent ground temperatures of less than $15^{\circ} \mathrm{C}$, which seems unlikely to be due only to surface temperature changes for this time of the year and this hour of the day. Moreover, occurrence of these cold points is generally associated with the presence of cirrus clouds in the region. If we now consider the points reaching their minimum VIS value or their minimum VIS value +1 count, $43 \%$ correspond to apparent ground temperatures of less than $15^{\circ} \mathrm{C}$, undoubtedly representing thin high clouds. This stresses the sensitivity of VIS threshold methods to the choice of the threshold.

Looking now, for each pixel, at the VIS values corresponding to maximum IR radiances and comparing them to the absolute minimum VIS values, we found that, over land, $27 \%$ of the values are different between the two cases (for radiometric resolution of $2^{\circ} \mathrm{C}$ ). If we further degrade the radiometric resolution, this percentage remains high $(18 \%$ for radiometric resolution of $6^{\circ} \mathrm{C}$.) Among these points, only $0.7 \%$ show large differences. The examination of the images shows that these $0.7 \%$ are associated with cloud shadows (Fig. 2). The greatest part of the points (26\%) different between the two images show differences of only one VIS count. This one VIS count difference can be attributed to a digitalization problem, radiometer noise, or a change during the period of solar zenith angle. However, a digitalization problem, radiometer noise and a change in the solar zenith angle cannot always explain these differences which occur sometimes in well-defined areas. A day-by-day examination of these areas shows that their pixels do not reach their minimum visible value on the warmest day but on a colder and cloudy day [maybe due to a higher water vapor content of the atmosphere, which can reduce the apparent albedo while reducing the apparent temperature (Koepke, 1982)]. 

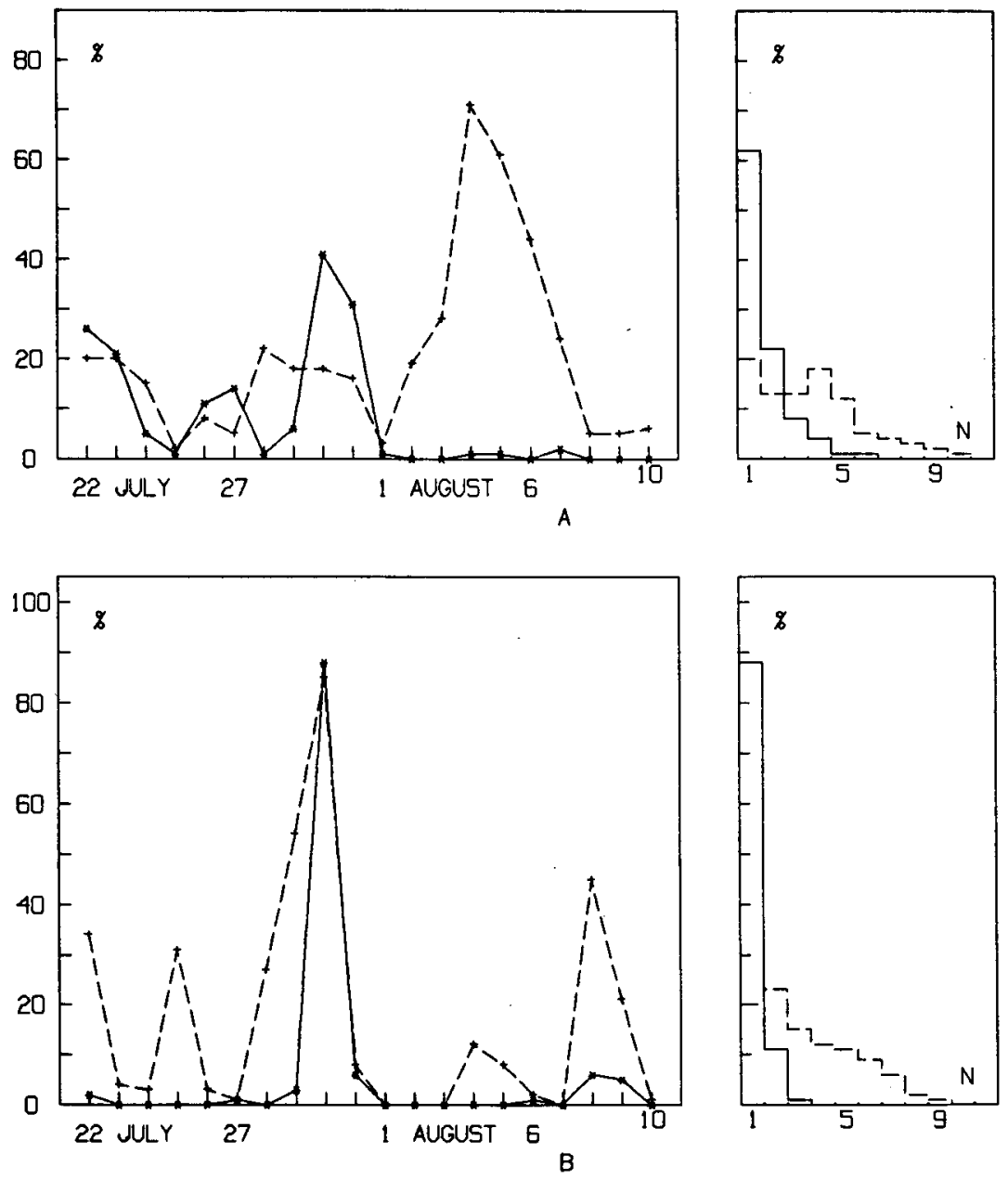

FIG. 6. (a) (left) Percentage of pixels reaching their maximum value in IR (full line) and their minimum value in VIS (dashed line) per day, for the Mediterranean region (region 1) (VIS range: 64 levels, IR range: 128 levels). (right) Percentage of pixels reaching their maximum IR value or their minimum VIS value $N$ times during the 20 days period. (b) As in Fig. $6 \mathrm{~A}$, except for the Paris region (region 4).

In some places over sea, the VIS image corresponding to the maximum apparent temperature is brighter than the minimum VIS image. This is due to the occurrence of a Saharan dust event: the dust increases the VIS reflectivity, and, as it is associated with an air mass warmer than the sea surface temperature, contributes to an increase of the IR radiance.

To summarize, from the minimum VIS composite image to the relative minimum VIS composite image one pixel value does not change by more than one count, except when the cloud shadows can be seen by the satellite over land, or when warm dust is present over the sea surface. These images seem, then, wellrepresentative of the surface reflectivity properties. However, VIS thresholding over land will not allow an accurate determination of cloud cover: a one-count threshold is not sufficiently high to be sure of the presence of cloud. On the other hand, it has also been shown that when the value of a pixel reaches its minimum, that does not always mean the absence of cloud.

The infrared channel may help for solving the ambiguities, but the time variability of the apparent surface temperature does not always allow a proper cloud/no cloud separation, specially for low clouds. It would then be useful to introduce the spatial VIS and IR local variabilities, which should be more constant in the absence of clouds than the VIS and IR radiances, and very different in the presence of clouds.

\section{Visible-infrared histograms-time-cumulative his- tograms}

After this pixel-by-pixel study of the surface properties, including their temporal variability and the surface/cloud separation based on composite image techniques, we focused on also obtaining the characteristics 
of the main kinds of clouds passing over the region during the 20 days by some kind of compositing.

In a first step, the repartition functions (or 2-d histograms) of the pixels of the studied region in the (VISIR) space were constructed for each day (Fig. 7). On this histogram series, the same characteristic shapes as described by Platt $(1981,1983)$ appear:

- A large spreading of the points along the IR axis with small VIS values is associated with cirrus clouds with large emissivity variations $(22,26,27,29,31 \mathrm{July}$ and 8 August). The minimum apparent temperature of these cirrus clouds is steady over the period.

- A simultaneous increase of the reflectivity (VIS counts) and decrease of the apparent temperature (IR counts) corresponds to days with presence of middle or middle-low level clouds, presenting some emissivity variations or producing partial coverage of many pixels (24 July and 3, 6 August).

- A very small variation in the VIS channel associated with a relatively weak variation of the IR (small reflectivity, high temperature) characterizes a clear sky day (such as 30 July) when very few clouds are present. This histogram is very similar to the one of the minimum VIS and maximum IR images constructed from the composite images of section 2.

- The presence of very low clouds is detected from an excursion in relatively bright values of points with a relatively warm apparent temperature (22 and 29 July).

- Regions of the histograms with large variations of the IR counts with a relatively high VIS value are characteristic of multiple layers. For example, for $28 \mathrm{July}$ and 2, 7 August, cirrus clouds are present together with medium-low-level clouds.

The examination of these series of histograms allows, thein, at first, a qualitative evaluation of what happens during the 20 days period; for example it can be deduced easily that the first part of the period is characterized mainly by the presence of cirrus, whereas the later part shows a denser cloud coverage, mainly with medium-low-level clouds.

The time variation of the maximum temperature, already discussed in section 2, appears again clearly, but cannot either be attributed preferentially to real changes of surface temperature or to the presence of clouds. For particular days, however, (1, 2, 3 August), the low-maximum temperature is clearly associated with a very dense cloud coverage. In these cases, this maximum is not representative of the surface, which is warmer. In this time series, generally, the real surface temperature variations remain far smaller than temperature variations due to the presence of clouds.

It appears, then, possible from this histogram time series to investigate some of the following mean properties of clouds during the period:

- stability of the histogram shapes associated with particular cloud types;

- relative position of the shapes corresponding to different cloud types occurring on different days and problem of multiple cloud layers;

- attribution to a particular cloud type of points which are not clearly associated with typical shapes on individual histograms;

Therefore, we condensed the information contained in each of the histograms into a single histogram, by constructing a cumulated histogram on the 20 days (Fig. 8 ). Figure 8 corresponds to the Paris region (region 4) whereas a schematic histogram corresponding to the Mediterranean region (region 1) is shown in Fig. 9a.

Clouds, which present a large variability in temperature and reflectivity, are spread along three axes representative of the preferential levels of cloud formation during the period (Fig. 9a). Each level of cloud forms a kind of "branch" starting from the brightest and apparently coldest points of the layer and converging to a common area. The surface pixels are condensed in the warmest part of this common area; however, due to the spatial and temporal apparent temperature variability of the surface, this warmest part also contains some pixels polluted by thin or partial coverage clouds.

To understand better the meaning of these branches, these experimental histograms were compared to theoretical curves relating apparent temperature to VIS reflectivity for different cloud layers. Such curves have been computed by Platt (1983) and by Arking and Childs (1985) from a radiative transfer model through cloud layers and are shown in Fig. 9b. The similarity between the theoretical diagrams and the experimental histograms indicates clearly that the spreading of the cloudy pixels along the "branches" can be interpreted

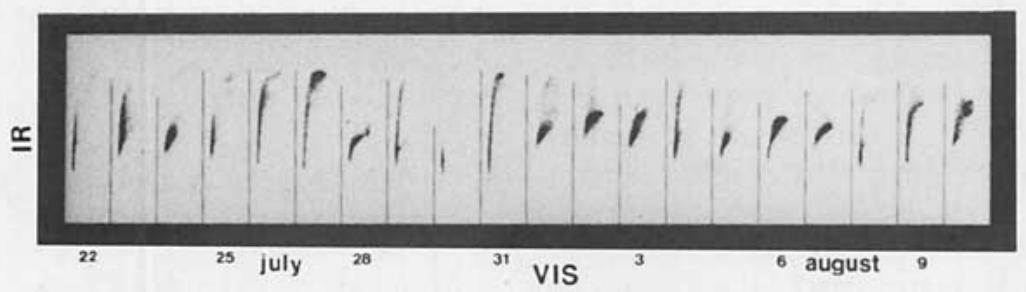

FIG. 7. Series of VIS-IR histograms for the period 22 July-10 August for region 4. The IR is on the vertical axis with cold upwards and VIS on the horizontal axis with bright to the right. 


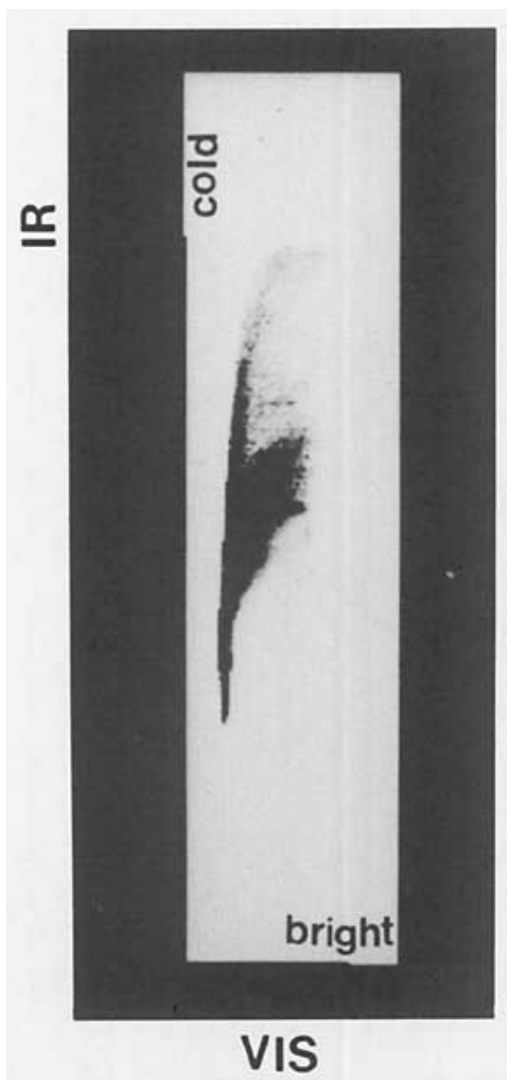

FIG. 8. Cumulated histogram for the whole period constructed from the series of Fig. 7 .

as due to the variations of the physical properties of the clouds (optical thickness and emissivity) or of the partial cloud coverage inside the pixels. The inclination of the different branches is a function of the altitude of the cloud layer involved.

These considerations are very useful for understanding the structure of the histograms, but it is still not possible to do an unequivocal correspondence between a particular point of the histogram and a particular cloud with determined physical properties. The most striking problem results from the presence of several kinds of clouds on the same diagrams, forming branches which overlap at the IR end nearest of the surface cluster(s) and also overlap part of the surface cluster(s) themselves. The points of this region form the "common area" which is defined in the schematic histogram of Fig. 9a. In this area, a pixel cannot be attributed to a specific layer only from its (VIS-IR) spectral signature. However, inside this common area, pixels with similar spectral characteristics, but belonging to different layers will not have the same spatial or textural characteristics in the corresponding VIS or IR image; for example, adjacent pixels covered by low or middle clouds on the image may have very different VIS values whereas their IR values will be similar; coverage by thin cirrus will produce the reverse behavior.
These textural properties or features can be quantified in different ways. Parikh $(1977,1980)$ computed a edge strength per unit area in the IR image. Coakley and Bretherton (1982) introduced the local IR standard deviation computed on small image areas. Tsonis (1984) associated with each VIS-IR spectral signature a mean spatial variance. In this analysis we used the local standard deviation in the VIS image and in the IR image computed for each pixel. These two local standard deviations constitute a good indicator of differences in spatial homogeneity between the cloud types (Belcour, 1985). Also, as it was noted in section 2, these standard deviations allow improved surface/cloud separation. They introduce a spatial information which may be complementary to the spectral information deduced from the spectral analysis. The information brought by the local variances is discussed in section 4.

\section{Variance images and associated histograms \\ a. VIS and IR variance images}

A variance image was constructed for each daily image and each of the two channels, VIS and IR: in these derived images, the value attributed to each pixel is the local standard deviation (lsd) in the $3 \times 3$ neighborhoods of the pixel (i.e., the standard deviation computed from the nine values centered on the pixel). Examples of these images for 22 July are given in Fig. 10 . On this particular image (panel a: VIS-panel b: IR) the coverage over France consisted essentially of high convective clouds, cirrus, and low stratiform clouds in the southwest. On the associated infrared variance image (panel d), the edges of convective clouds are welldefined by very high variances, whereas the variance is far smaller inside these "contours." Cirrus correspond to areas of high variances and low stratiform clouds to areas of low variances. On the associated VIS variance image (panel c), edges of convective clouds still present large variances, but the summits themselves have relatively higher variances than in infrared; contrary to the IR variances, low VIS variances are associated with cirrus clouds and relatively high variances with low stratiform clouds. In order to better understand the information contained in these two derived images, and its relation with the information contained in the VIS and IR images, we have constructed series of different kinds of histograms: infrared versus infrared variance (Coakley and Bretherton 1982, Coakley and Baldwin 1984), visible versus visible variance, and infrared variance versus visible variance.

\section{b. Infrared-infrared variances diagrams (Fig. 11a)}

These diagrams frequently show arch shapes, as described by Coakley and Bretherton. The ensembles of low variance points, or arch feet, appear clearly, allowing the determination of the apparent temperature of the different homogeneous zones of the image (either 

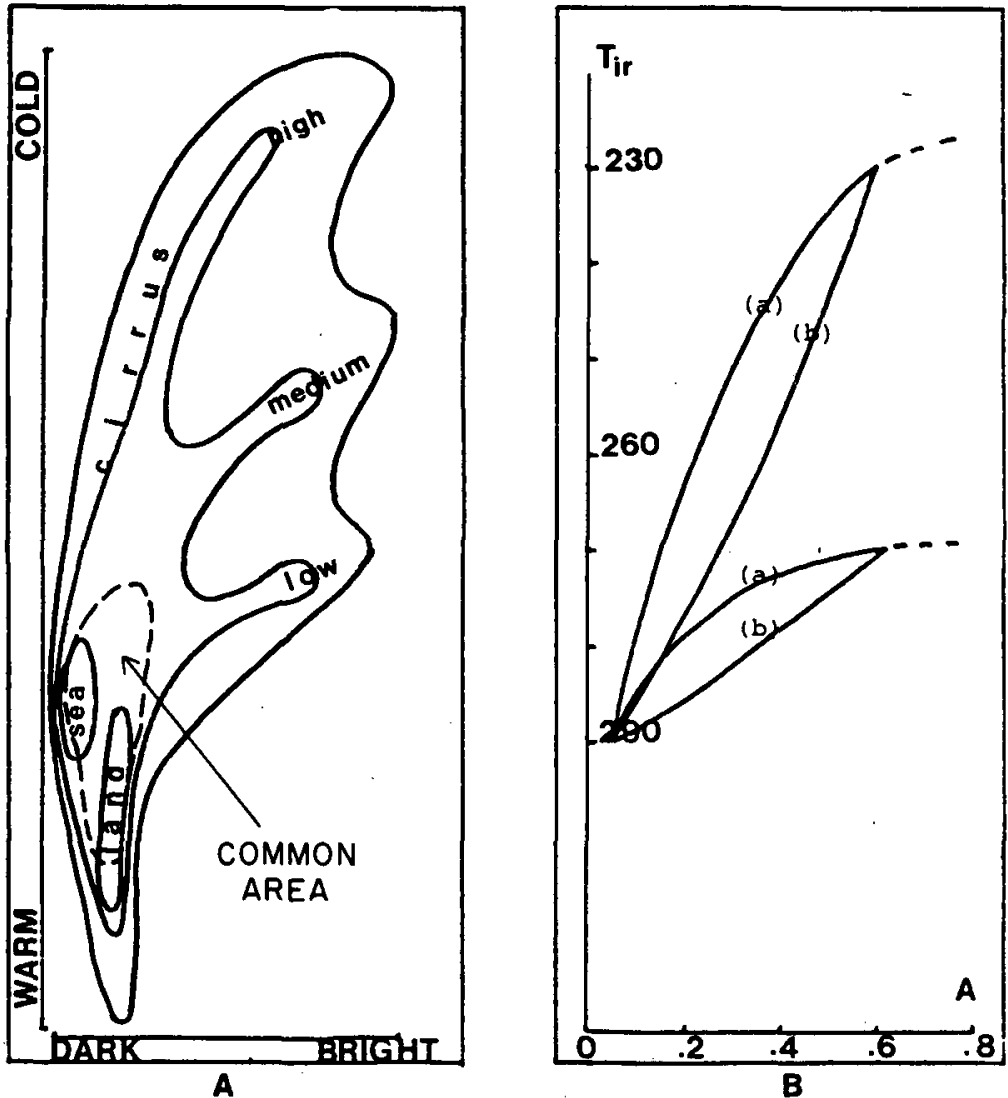

FIG. 9. (a) Interpretation of the cumulated histogram obtained in region 1. (b) Theoretical shapes of temperature-albedo curves obtained by Platt, for two layers of clouds with varying optical thickness (curves a) and partial coverage of the pixels (curves b).

surface or clouds). The arch feet are linked by the arch "bodies" which are composed of pixels with intermediate temperature values and with larger standard deviations. Coakley and Bretherton explain these arch bodies as due to a partial coverage of the pixels with the elements constituting the feet of the arches (in simple cases, partial coverage with one surface type and one cloud type). The examination of the present series shows that these points can be due to emissivity variations inside a single cloud; that is especially clear in the case of cirrus. Finally, arches are due to the variation of the effective cloud coverage $N \epsilon$ where $N$ is the partial coverage and $\epsilon$ the emissivity. In some cases (small thick clouds), the variation of $N$ is predominant and in others (extended thin clouds), the variation of $\epsilon$ is the largest.

\section{c. Visible-visible variances diagrams (Fig. 11b)}

A very well defined arch foot appears generally for the low visible values, corresponding to the surface pixels. At the other extreme, feet corresponding to homogeneous cloud zones are far less common than in the infrared-infrared variance diagram (Fig. 11a). This can be attributed to two causes:
- Even when the emissivity $\epsilon$ remains equal to 1 , resulting in homogeneous areas on the IR image for a cloud layer, the optical thickness $\tau$ can still vary, resulting in different values of the visible light reflected towards the satellite.

- Geometrical structure of the cloud surface can produce much more variations in the reflected sunlight than in the IR radiance of the cloud layer. However, the use of these diagrams may allow a good determination of the surface properties and of different cloud layers if locally the layers entirely cover the pixels and are very thick.

\section{d. Infrared variances-visible variances histograms (Fig. 11c)}

Some of these histograms show a spreading of points along the IR variance axis, with low VIS variances, corresponding to a cirrus coverage. This spreading can be compared to the one which was observed along the infrared axis of the VIS-IR histograms (Fig. 7) for the same clouds $(22,25,26,29$ July and 8 August). Others, such as for 24 July and 1, 3 August show correlated variations of visible and infrared standard deviations; 


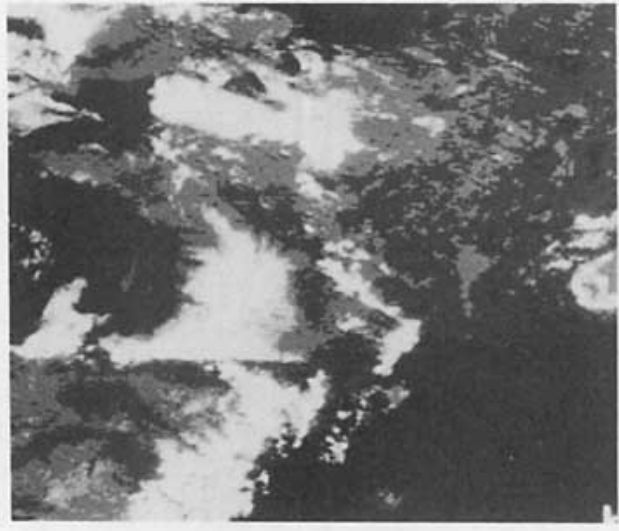

A

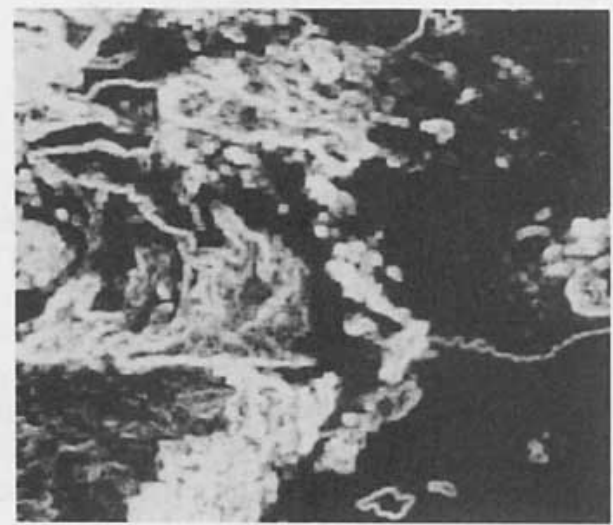

c

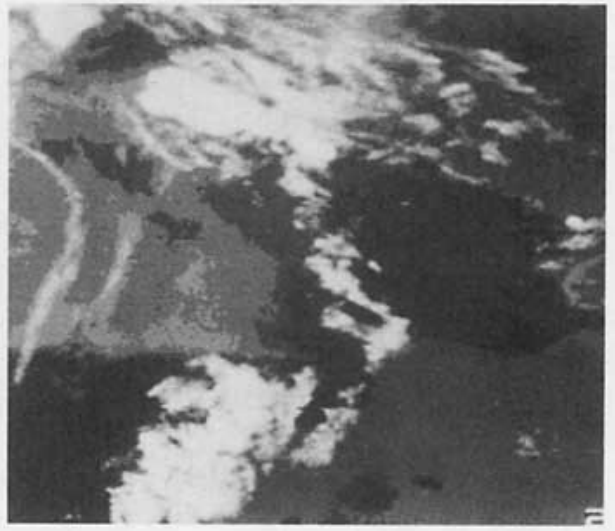

B

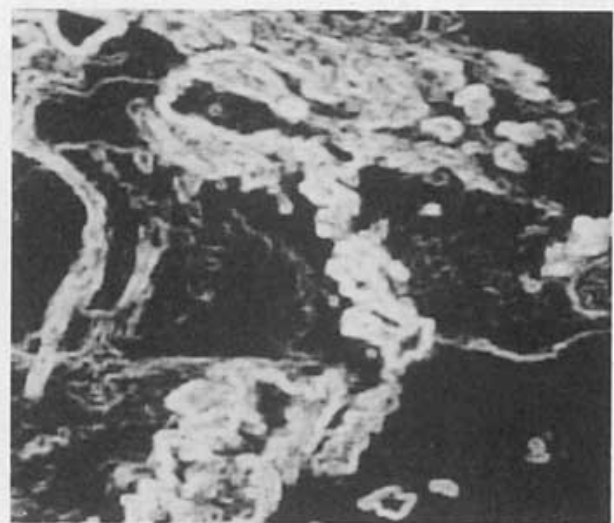

D

FIG. 10. VIS and IR images ( $a$ and b, respectively) for 22 July, and corresponding variance (c and d).

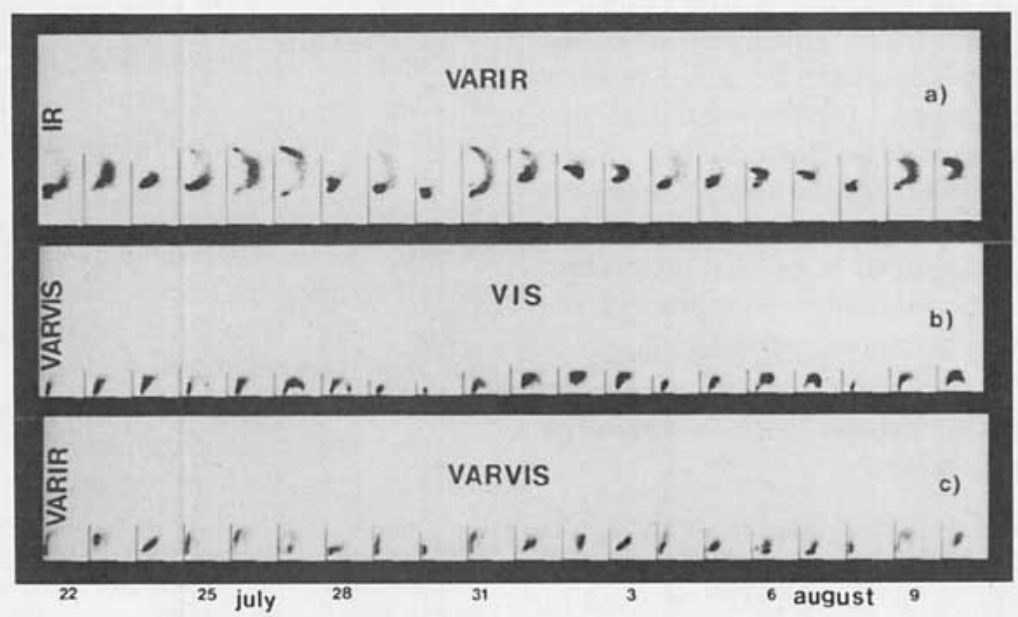

FIG. 11. As in Fig. 7 but for (a) infrared-infrared variances histograms, (b) visible-visible variance histograms and $(c)$ infrared variance-visible variance histograms. 
they correspond to partial coverage by middle-lowlevel clouds.

The spreading along the VIS variance axis for relatively low IR variances observed on 28 July and 6,7 August is characteristic of a quasi-total coverage by middle-low-level clouds (due to the relation $\tau \cdot \epsilon$ as explained in section 4c). Other points appear also, with higher IR variances due to the presence of some partial coverage and/or cirrus. In the case of cirrus lying over low or middle clouds, as for 2 August, a spreading along the IR variances axis is associated with relatively high VIS variances.

\section{e. General interpretation of variance diagrams}

The empirical interpretation of these results can be summarized as follows:

$\begin{array}{lc}\begin{array}{c}\text { Low VIS variance, } \\ \text { low IR variance }\end{array} & \begin{array}{c}\text { Surface, homogeneous } \\ \text { thick clouds } \\ \text { Relatively low VIS } \\ \text { variance, high } \\ \text { IR variance }\end{array} \\ \begin{array}{c}\text { Cirrus over surface } \\ \text { Relatively high VIS } \\ \text { variance, low IR } \\ \text { variance }\end{array} & \begin{array}{c}\text { Quasi-total coverage by } \\ \text { middle-low clouds }\end{array} \\ \begin{array}{c}\text { High VIS and IR } \\ \text { variances, with } \\ \text { correlated varia- }\end{array} & \begin{array}{c}\text { Partial coverage by } \\ \text { tions }\end{array} \\ \end{array}$

According to this interpretation, IR variances-VIS variances histograms allow the distinction between partial cloud coverage, semitransparent clouds and surfaces which could not be separated on the VIS-IR histograms and were often gathered in the common area described in section 3 . The combination of the two kinds of information, which can be called spectral for the VIS and IR and spatial for the local standard deviations, may allow a better determination of the cloud types when the clouds form homogeneous layers as well as when they produce partial coverages of the pixels or present local variations in optical properties. A discussion of the test of a classification method using these four parameters, is presented next.

\section{Simultaneous use of spectral and spatial information in a classification scheme}

The basic algorithm (Dynamic Clusters method; Diday and Simon, 1980) used here to combine spectral' and spatial information is the same that was used in Desbois et al. (1982). However, in that paper, the algorithm was applied to the three Meteosat channels; visible $(0.4-1.1 \mu \mathrm{m})$, infrared $(10.5-12.5 \mu \mathrm{m})$ and water vapor $(5.7-7.1 \mu \mathrm{m})$. In the present study, the method combines spectral and spatial information. Moreover, it is not applied to individual images, but to cumulated data, as it was already discussed in Desbois and Seze (1984). Each pixel of a chosen image segment is represented by four parameters: two spectral ones (visible and infrared values) and two spatial ones (visible local standard deviation and infrared local standard deviation). First a 4D cumulative histogram is built up from pixels sampled in space and time on 10 of the 20 initial images of the series. ${ }^{1}$ The central concept of the method is that every surface or cloud type in the image segments is represented in this cumulated histogram by a compact subdomain of the histogram (or cluster). A cluster is characterized by its center of gravity (VIS, IR, LSD VIS, LSD IR) and variance in the histogram. The method does not require any a priori knowledge of the classes, but only a maximum number of classes (chosen as $K=15$ in the present case). To partition the histogram, the method uses an iterative process starting from randomly chosen clusters. After each iteration, a criterion decreases and a new partition is defined. The final partition is obtained when the iterations give always almost the same partition. However, this last partition is not unique, because the criterion converges toward a local optimum. The choice of the initial clusters has, then, an influence on the final partition. This constitutes one of the main limitations of this kind of technique, (Diday and Simon, 1980). Therefore, we made several random choices of the initial clusters (three or four). The partitions obtained are generally similar, and the one which seems the best, according to the criterion value, the number of classes left and the values of the center of gravities is chosen. Finally, each pixel of the 20 images is projected on the 4D histogram and is attributed to the class of which it is the nearest, for the chosen partition.

As the computation of the distance between the points is done according to the euclidian distance, the choice of the dynamic range of the different variables has an effect on the classification. In the present case, IR range has been kept to its initial 256 levels, VIS range has also been extended to 256 levels from its initial 64 values. The 1sd have been multiplied by 30 in order to get a compatible range. Table 1 lists the average and standard deviation of all the variables used, indicating their respective weight.

The classification has been applied to six regions, including those defined in Fig. 1. and covering West Germany, France, and South England. The size of these regions have been latitudinally constrained in order to avoid large spatial variations of surface or atmospheric

\footnotetext{
${ }^{1}$ These 10 days have been chosen from the histogram shapes in order to get a sample representative of the different cloud situations occurring during the period. The influence of the choice of the days on the resulting classification has been tested by making classification using the 10 first days, then the 10 last days of the period. Classes remain steady; however, some differences, due to the different sampling, appear: during the second period, there are less high clouds and the surface appears less often, producing corresponding less welldefined classes.
} 
TABLE 1. Average and standard deviations (in 8-bit counts) of the four parameters used in the classification, showing the range of variation of each one. The IR scale has been inverted for visualization purpose.

\begin{tabular}{lcc}
\hline \hline & Average & Standard deviation \\
\hline VIS & 73 & 31 \\
IR & 129 & 34 \\
VARVIS & 25 & 17 \\
VARIR & 47 & 20 \\
\hline
\end{tabular}

properties. Most of the results presented here concern a $40 \times 120$ pixels area centered on the Paris region (region 4), as it was the case for the previously presented histograms.

Nine classes have been found, whose centers of gravity parameter values are given in Table 2 . The identifications given to the classes are based on the following observations:

1) values of the different parameters for the centers of gravity,

2) projection of the points of each class on the VISIR histogram, and

3) comparison of the classified images to the corresponding original images.

First, we can separate the most homogeneous classes corresponding either to the surface or to well-defined cloud types:

- class 9: the most homogeneous spatially in VIS and IR, and also the warmest and the darkest, is characteristic of the surface;

- class 1: relatively homogeneous in both channels, but very cold and bright, corresponds to high thick clouds; and
- class 4: very homogeneous in IR with an intermediate temperature corresponding to total stratiform coverage with medium level clouds.

Among the classes with high local standard deviations, three groups can be separated:

- Classes 2, 6, 7 are characterized by their high IR lsd, and their high ratio IR lsd/VIS lsd, which denotes the presence of cirrus. Class 7 has a very low VIS lsd, and represents a thin cirrus coverage over ground or sometimes thin edges of middle level clouds. Classes 6 and 2 present larger VIS Isd and may correspond to thicker cirrus or to cirrus above other cloud layers.

- Classes 8 and 5 with VIS lsd of the same order than IR lsd correspond to more or less partial coverage by medium-low clouds.

- Class 3 is intermediate between these two groups and covers a large range of temperatures, although its percentage is not very high. It may correspond either to cirrus over other cloud cover or to high cloud partial coverage.

This first examination of the classification obtained with four parameters indicates that this method is efficient in separating a homogeneous surface class, total coverage cloud classes and thin cirrus versus partial coverage of the pixels. However, in order to test more objectively the effect of introducing VIS lsd and IR lsd parameters, we compared the results to the classification obtained by using only VIS and IR radiances. In this case, seven classes were obtained (Table 3 ). The IR and VIS values of their centers of gravity allowed an association with the classes obtained with four parameters. The percentage of surface detected during the whole period is $9.2 \%$ higher in the case of the two parameters. Less thin cirrus and partial coverage has

TABLE 2. Values of the centers of gravities in counts of the nine classes obtained with the four parameters (VIS-IR-VIS Isd-IR-lsd) classification. Albedo and apparent temperature correspond to the VIS and IR values and var. c. stands for class variance, which is an indicator of the dispersion of the class around its center of gravity. The last column gives the percentage of occurrence of the class during the whole period.

\begin{tabular}{rrrrrrrrrr}
\hline \hline C & VIS & IR & VIS lsd & IR lsd & var c & Alb & $T(\mathrm{~K})$ & $\%$ & Cloud cover* \\
\hline 1 & 151 & 207 & 33 & 31 & 120 & 0.72 & 229 & 3.2 & high thick cl \\
2 & 94 & 185 & 35 & 59 & 101 & 0.45 & 248 & 8.4 & cirrus \\
3 & 136 & 152 & 50 & 53 & 80 & 0.65 & 270 & 6.8 & $\begin{array}{c}\text { cirrus over other cl high cl } \\
\text { part cov } \\
4\end{array}$ \\
& 138 & 140 & 35 & 21 & 48 & 0.66 & 276 & 5.8 & mid lev stratiform cl \\
5 & 97 & 134 & 46 & 47 & 48 & 0.46 & 279 & 10.9 & mid low cl part cov \\
6 & 72 & 146 & 38 & 71 & 115 & 0.34 & 273 & 10.6 & cirrus \\
7 & 57 & 123 & 15 & 66 & 30 & 0.27 & 285 & 12.5 & cirrus \\
8 & 73 & 119 & 32 & 39 & 29 & 0.35 & 287 & 14.3 & mid low cl part cov \\
9 & 52 & 99 & 10 & 27 & 13 & 0.24 & 297 & 27.3 & surface \\
\hline
\end{tabular}

* $\mathrm{cl}=$ clouds; mid = middle; part cov = partial coverage 
been detected. The distinction between cirrus and noncirrus classes has not been possible in several cases.

The usefulness of the introduction of the VIS lsd parameters was also tested in the same way, by computing a three-parameter classification using VIS, IR and IR Isd only. In this case, nine classes were obtained (Table 4) and their centers of gravity parameter values are close to those obtained with four parameters. Table 5 shows that the correspondence between the two classifications for the whole period is good. But a day by day examination of the results shows some differences, mostly for the separation between partial coverage pixels and ground. The percentage of pixels classified as ground in the three-parameter classification and as partial coverage in the four-parameter classification can be up to $7 \%$ for some days when there is a large proportion of partial coverage in the four-parameters classification. There is also a difference in the definition of the class of thin cirrus over land. The small differences existing between other classes are due to light changes in the separations of nearby classes.

Finally, the general stability of the three-parameter classification results versus those obtained with four parameters shows the predominance of the IR Isd. However, in the case of very partial coverage or very thin cirrus layer, VIS lsd yields additional information despite the small weight which was given to it at the initial state of the classification.

Addition of the local standard deviations in a clustering technique appears to improve the results, for cloud/surface separations as well as for discrimination between partial coverage of the pixels and semitransparent clouds. However, the results obtained by this kind of technique are based on statistical considerations, and it is useful to see how they compare with results of threshold techniques, which work on a pixelby-pixel basis. It is also important to assess the validity of the results relative to conventional measurements. Discussion of these comparisons follows. A comparison with threshold techniques is presented in section 6 , and the possibility of verifications from other data is discussed in section 7 .

\section{Comparison with a simple threshold technique}

The clustering technique used here is based on statistical spatial and temporal properties of the data over a region. Therefore, the results are sensitive to the spatial and temporal variations of the regional surface properties. On the other hand, threshold techniques, because they work on a pixel-by-pixel basis, are theoretically insensitive to spatial variations and sensitive to temporal ones. Indeed, surface radiances maps are frequently constructed with techniques similar to the ones used in section 1 of this paper, and are representative of a time period and not of a particular day.

The comparison discussed here concerns the "clear sky" identification (complementary to the total cloud coverage). We used as reference maps the minimum visible and maximum infrared composites built on 19 days (section 1) and computed the percentage of totally cloud free pixels in the Paris region based on different thresholds:

\section{Visible $\quad 0,1,2$ VIS counts (64 count range) i.e., $2,4,6 \%$, approximately, in al- bedo \\ Infrared $\quad 0,2,4,8,12$ IR counts (256 count range) i.e., $1 / 2,1,2,4,6^{\circ} \mathrm{C}$, approxi- mately, in apparent temperature
Combined $(0,2),(1,4),(1,8),(2,12)$ VIS or IR visible and counts; i.e., $2 \%$ or $1{ }^{\circ} \mathrm{C}, 4 \%$ or $2^{\circ} \mathrm{C}$, infrared $\quad 4 \%$ or $4^{\circ} \mathrm{C}$ and $6 \%$ or $6^{\circ} \mathrm{C}$.

A pixel will be counted as cloudy if its radiance is greater than the sum of the reference surface radiance and the threshold value. The results of these thresholdings are shown in Table 6, and Fig. 12 for each of the 20 days of the period, and they are compared to the percentage of surface found by the clustering method (discussed in section 5). Analysis of this table shows that

- for a 0 VIS count threshold, the percentage of sur-

TABLE 3. As in Table 2 but for a two-channel (VIS-IR) classification (seven classes).

\begin{tabular}{|c|c|c|c|c|c|c|c|c|c|}
\hline $\mathrm{C}$ & VIS & IR & VIS Isd & IR Isd & $\operatorname{var} \mathrm{c}$ & Alb & $T(\mathrm{~K})$ & $\%$ & Cloud cover* \\
\hline 1 & 159 & 207 & & & 28 & 0.76 & 229 & 2.0 & high thick cl \\
\hline 2 & 104 & 191 & & & 29 & 0.50 & 243 & 7.1 & $\begin{array}{l}\text { ci, ci above other high } \\
\text { clouds part cov }\end{array}$ \\
\hline 3 & 71 & 155 & & & 14 & 0.34 & 266 & 11.4 & cirrus \\
\hline 4 & 146 & 139 & & & 8 & 0.70 & 271 & 7.0 & mid lev stratiform $\mathrm{cl}$ \\
\hline 5 & 109 & 141 & & & 6 & 0.52 & 276 & 12.9 & mid low cl part cov \\
\hline 6 & 75 & 159 & & & 2 & 0.36 & 285 & 23.1 & cirrus \\
\hline 7 & 54 & 123 & & & 0 & 0.26 & 295 & 36.5 & cirrus \\
\hline
\end{tabular}

* $\mathrm{cl}=$ clouds; mid = middle; part cov = partial coverage; $\mathrm{ci}=$ cirrus 
TABLE 4. As in Table 2 but for a three-parameter (VIS-IR-IR lsd) classification (nine classes).

\begin{tabular}{|c|c|c|c|c|c|c|c|c|c|}
\hline $\mathrm{C}$ & VIS & IR & VIS lsd & IR Isd & $\operatorname{var} \mathrm{c}$ & Alb & $T(\mathrm{~K})$ & $\%$ & Cloud cover* \\
\hline 1 & 154 & 208 & & 30 & 62 & 0.73 & 288 & 3.2 & high thick cl \\
\hline 2 & 103 & 191 & & 55 & 80 & 0.49 & 243 & 8.4 & cirrus \\
\hline 3 & 148 & 155 & & 51 & 29 & 0.71 & 267 & 6.3 & $\begin{array}{l}\text { cirrus over other cl high } \\
\text { cl part cov }\end{array}$ \\
\hline 4 & 136 & 139 & & 22 & 22 & 0.65 & 277 & 5.7 & mid lev stratiform cl \\
\hline 5 & 105 & 141 & & 49 & 34 & 0.50 & 276 & 10.9 & mid low cl part cov \\
\hline 6 & 72 & 159 & & 70 & 48 & 0.34 & 265 & 10.5 & cirrus \\
\hline 7 & 59 & 123 & & 68 & 16 & 0.28 & 285 & 12.7 & cirrus \\
\hline 8 & 78 & 121 & & 40 & 11 & 0.37 & 286 & 13.9 & mid low cl part cov \\
\hline 9 & 53 & 100 & & 28 & 8 & 0.25 & 296 & 28.5 & surface \\
\hline
\end{tabular}

* $\mathrm{cl}=$ clouds; mid = middle; part cov = partial coverage

face is nearly always smaller than with the classification, except for 2 days when much thin cirrus is present;

- for a 1 VIS count threshold, the percentage of surface is nearly always greater than with the classification, except for 5 days when much much-smaller partial coverage occurs; and

- for a 2 VIS count threshold, the cloud cover is always underestimated.

It appears that the difference between results obtained with two different thresholds ( 0 and 1 count) is greater than between the classification and results obtained with each of these thresholds. The 1-count threshold generally gives results closer to the classification, except in presence of cirrus clouds when the percentage of surface is overestimated.

In order to better account for these cirrus clouds, an infrared threshold was applied. In this case, the threshold has to be as high as $6^{\circ} \mathrm{C}$ to get the best correspondence with the classification; all the lower thresholds produce an underestimation of the clear sky pixels relative to the results of the classification. It appears that the construction of an IR reference image from too long a series of images leads to an overestimation of the cloud cover, as the warmest day is taken as indicative of the surface radiance for the whole period. Further tests were conducted from shorter series: the percentage of "clear sky" increases, and even becomes too large for very short series ( 5 days), when the composite image remains polluted by the persistence of clouds over some areas. On the other hand, the percentage of very thin cirrus over land may have been underestimated on some days by the classification.

For the case of combined IR and VIS thresholds, (i.e., a pixel is considered cloud free if VIS and IR thresholds are not exceeded), the separation by the infrared is predominant and the results are much like those obtained with the IR threshold only.

It appears that the four-parameter classification fits reasonably well (for the total cloud cover) with the VIS threshold when there are few thin cirrus, and with the IR threshold when the maximum temperature for the particular day studied is very close to that of the composite IR picture. However, the classification presents

TABLE 5. Correspondence between three-parameter and four-parameter classifications. The numbers in the table are the percentages of pixels classified simultaneously in class $i$ of the three-parameter classification and $j$ of the four-parameter classification.

\begin{tabular}{|c|c|c|c|c|c|c|c|c|c|c|}
\hline \multirow[b]{2}{*}{$j$} & \multicolumn{9}{|c|}{$i$} & \multirow[b]{2}{*}{ Cloud cover* } \\
\hline & 1 & 2 & 3 & 4 & 5 & 6 & 7 & 8 & 9 & \\
\hline 1 & 1 & 0 & 0 & 0 & 0 & 0 & 0 & 0 & 0 & high thick cl \\
\hline 2 & 0 & 4 & 0 & 0 & 1 & 2 & 0 & $\mathbf{0}$ & 0 & cirrus \\
\hline 3 & 0 & 0 & 7 & 1 & 3 & 0 & 0 & 0 & 0 & cirrus over other $\mathrm{cl}$ high $\mathrm{cl}$ part cov \\
\hline 4 & 0 & 0 & 1 & 9 & 0 & 0 & 0 & $\mathbf{0}$ & 0 & mid lev stratiform cl \\
\hline 5 & 0 & 0 & 0 & 0 & 13 & 0 & 0 & 4 & 0 & mid low cl part cov \\
\hline 6 & 0 & 0 & 0 & 0 & 0 & 7 & 3 & 0 & 0 & cirrus \\
\hline 7 & 0 & 0 & 0 & 0 & 0 & 0 & 7 & $\mathbf{0}$ & 0 & cirrus \\
\hline 8 & 0 & 0 & 0 & 0 & 0 & 0 & 1 & 16 & 1 & mid low cl part cov \\
\hline 9 & 0 & 0 & 0 & 0 & 0 & 0 & $\mathbf{0}$ & $\mathbf{0}$ & 18 & surface \\
\hline
\end{tabular}

* $\mathrm{cl}=$ clouds; mid = middle; part cov = partial coverage 


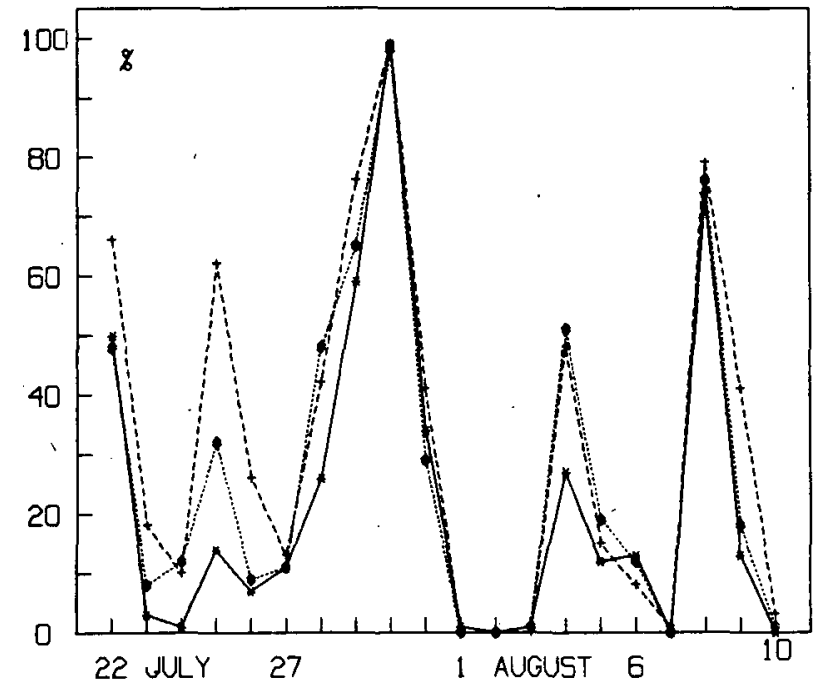

FIG. 12. Percentage of clear sky obtained for each day by 0 -count VIS threshold (long dashed line); 12-count IR threshold (solid line); and four-parameter classification (short dashed line).

the supplementary advantage of determining automatically the cloud-no cloud separation. In this process, the local variances play a role which allows the differentiation of clouds by another way than separation in the VIS and IR domains. It thus compensates for the problems due to temporal variability of surface apparent temperatures. This characteristic is very important in light of the sensitivity of cloud separation to the choice of the thresholds. Moreover, the VIS threshold which gives the best correspondence with the classification seems to be either 0 or 1 counts, which corresponds to the considerations of section 1 relative to the definition of the surface properties from VIS composite images. While there is no absolute argument to prove that the four-parameter classification is the best, we have shown in section 5 the usefulness of these four parameters for the separation of cloud types; the separation obtained by the classification is the more "natural" accounting for these data. An improvement which could be added to this kind of approach would be a tuning of the relative weight given to each of the parameters. For example, an increase of the weight of the IR variance could improve the cirrus-clear sky separation. Another change which should be tried is to adapt the method to the particular shape of cloud and surface clusters which are observed on the histograms. This could be achieved by changing the definition of the distance used in this version of the Dynamic Clusters technique.

\section{Comparison of the results of the classification with independent measurements}

It would be useful to compare the results of the classification to independent measurements of the cloud cover. This is a common problem for all the satellite cloud classification techniques, (Parikh and Ball, 1980), (Minnis and Harrison, 1984), and particularly for the validation of the ISCCP results. This comparison is beyond the scope of this paper as large-scale satellite classifications are difficult to compare to local conventional cloudiness estimations on a daily basis. Big experiments or programs (FIRE, for example) which have been planned to intensively study particular cloud types (stratocumulus, cirrus) in conjunction with satellite

TABLE 6. Time evolution of the percentage of "clear sky" during the whole period according to different ways of determination. First group: dynamic cluster classification with four, three and two parameters; Second group: separation with different visible thresholds (six-bit counts); Third group: separation with different infrared thresholds (eight-bit counts); Fourth group: combined VIS and IR thresholds.

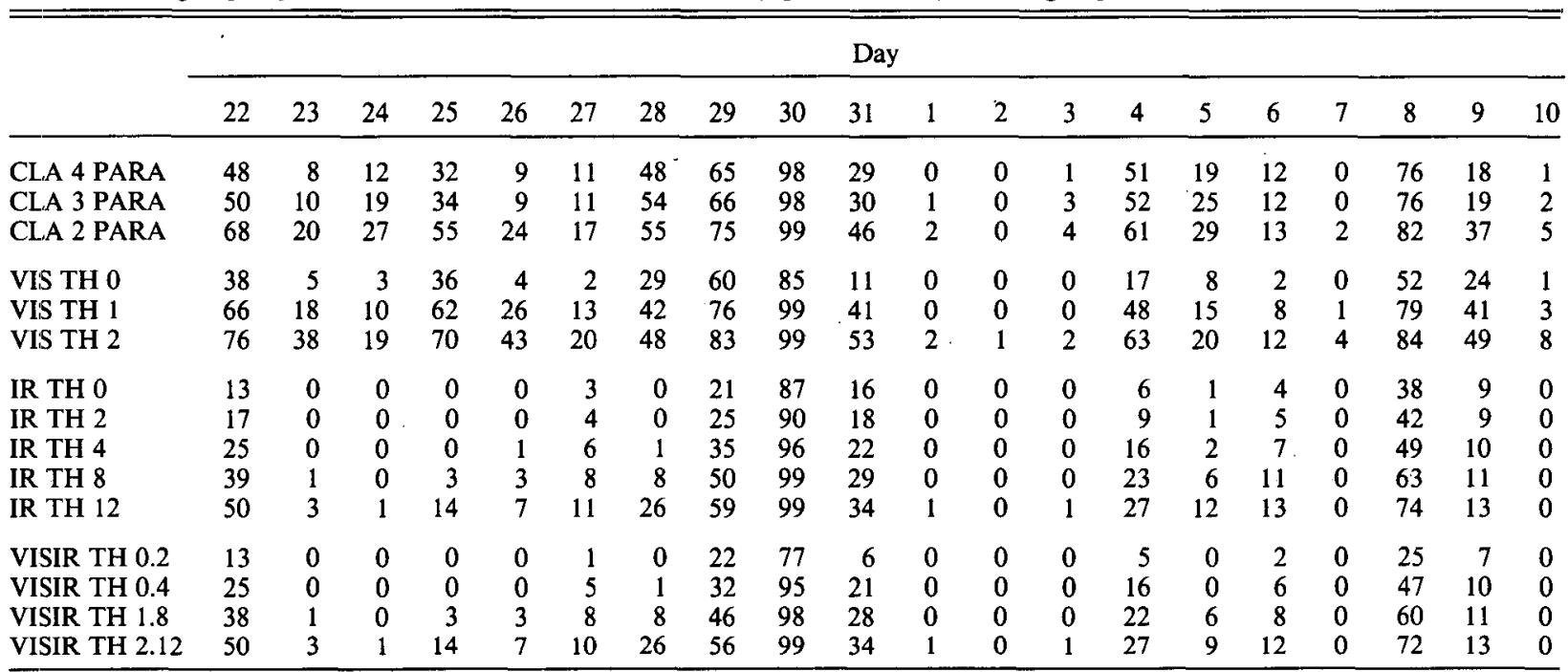


studies, will be the best tool for such validations in the future.

A comparison of the results of the classification used here with conventional ground measurements of cloudiness (in oktas) was presented by Seze et al. (1986). The results are in reasonable agreement for the total cloud cover. Comparison between cloud classes is much more difficult due to the completely different ways of observing the clouds: from above at large scale for the satellite, and from below with a restricted field of view for the ground observers.

Results of the classification can also be compared to conventional manual nephanalyses made in meteorological services. That kind of comparison cannot be called a "validation," as the results are deduced from the same kind of data in both analyses; but it can bring information on the class separation differences and on the usefulness of the automatically separated classes in regard to the conventional classes of the nephanalysts. For the period analyzed here, two kinds of nephanalyses were available from the "Centre de Meteorologie Spatiale" of Lannion (France): A large scale analysis from Meteosat at noon and more detailed ones from AVHRR images of NOAA satellites in the early afternoon. For the main cloud systems, the level of agreement between the two nephanalyses is good; differences which appear can be generally attributed to the nonsimultaneity between the AVHRR image and the Meteosat image, or sometimes to the lack of details of the Meteosat nephanalyses.

A visual comparison between these two nephanalyses and the classified image has been made for each day. A nephanalysis for a particular day (22 July) and the corresponding classification from the present study is presented in Fig. 13. As expected, the global structure of the cloud systems is similar in nephanalyses and classified images. The comparison of cloud types defined by the analyst as $\mathrm{Sc}, \mathrm{Cu}$, Ac (often associated with $\mathrm{Ci}$ ), $\mathrm{Ci}$, and $\mathrm{Cb}$, and defined by the classification as thin clouds, partial coverage by middle-low-level clouds, middle-level clouds, cirrus, cirrus overlying another layer, and high clouds, shows

- For $\mathrm{Sc}$ and $\mathrm{Cu}$ coverage detected by the nephanalyst, the classification gives either medium clouds or partial coverage by medium or low clouds depending on the coverage: dense cloud coverages by $\mathrm{Sc}$ or $\mathrm{Cu}$ are mainly classified as medium clouds, low coverages being attributed to partial coverage cloud classes. This often results in a mixing of different cloud classes in the classification charts, corresponding to more or less dense coverage regions. In some cases on the extreme edges of these $\mathrm{Sc}$ and $\mathrm{Cu}$, the class corresponding to thin clouds can be found. Are these clouds really optically thin, or is it an effect of partial coverage and/or surface temperature variations? This cannot be answered here, and needs surface validations.

- For Ac reported by nephanalysis, classification
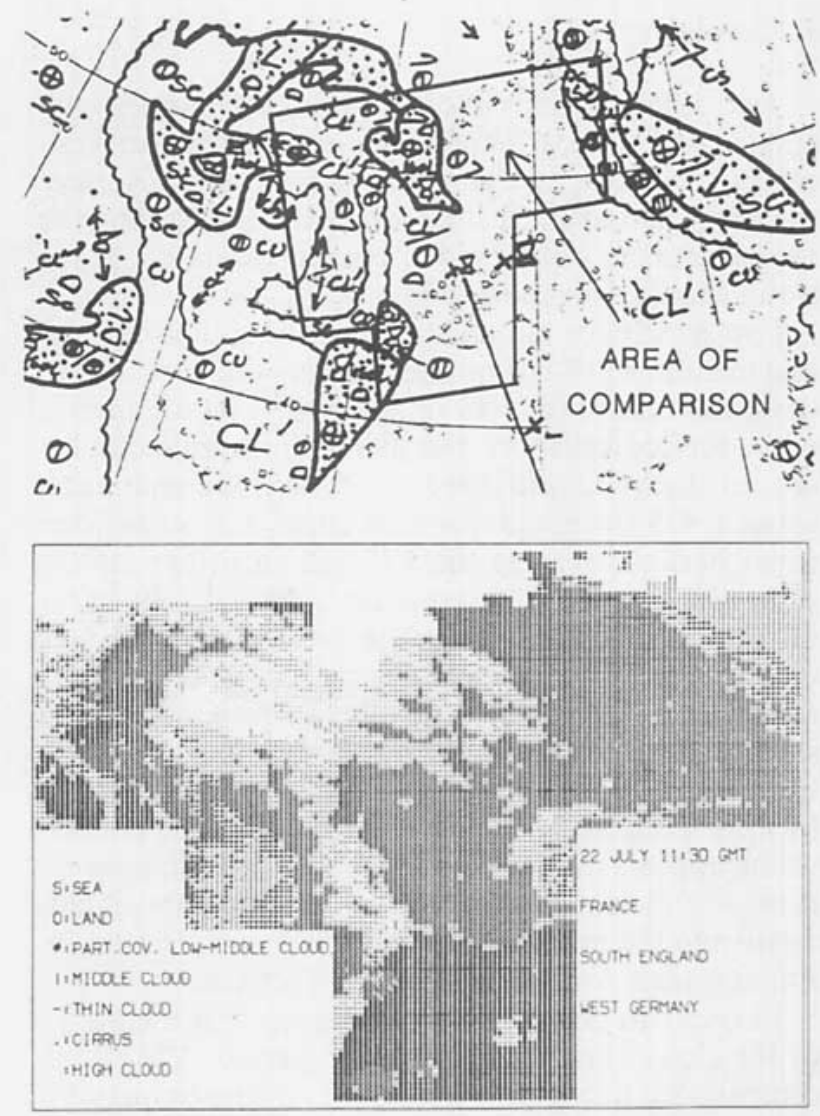

FIG. 13. (Top) The AVHRR nephanalyses for 22 July (1315-1454 UTC). (Bottom) The four-parameter classification obtained for 1150 UTC 22 July.

detects high clouds, middle clouds and sometimes cirrus lying above low or middle clouds. For dense coverage of Ac associated with cirrus, the classification gives high clouds, thick cirrus, or cirrus lying above low or middle cloud.

- Cirrus of the nephanalyst are classified as cirrus, except when they are very thick, and are then classified as high thick clouds. All $\mathrm{Cb}$ are also attributed to this last class.

- It appears, then, that a systematic correspondence can be found between the manual nephanalysis and the classification, despite the differences of approaches between the two techniques: The analyst attributes a name to a cloud deck, (as $\mathrm{Sc}, \mathrm{Cu}, \mathrm{Ac}, \mathrm{Ci}$ ) by using his experience of cloud systems, and relies more on cloud structures at different scales than on the absolute VIS and IR radiances values. The classification attributes a name to each pixel according to its VIS and IR radiances values, taking into account only a local cloud structure parameter, the local lsd in the images. For one cloud deck, this can lead to several class names; these class names are representative of the cloud top level while nephanalysis names are representative of general cloud types, whose level is generally associated with the cloud base level. 


\section{Conclusion}

Two different kinds of compositing techniques were applied on a time series of Meteosat pictures over western Europe. The first aimed to determine the surface radiances and to study their temporal stability and the second aims to obtain radiances representative of the cloud classes over the period.

For the surface radiances study, the minimum VIS and maximum IR composite images were used. On 20 days, the minimum VIS image is well-representative of the surface radiances, but these minimum radiances are not always adapted for a cloud/no cloud separation using a VIS threshold: for thin cirrus and sometimes small partial coverage the VIS radiances can be the sarne as the surface VIS radiances. Alternatively, in the maximum IR composite image on 20 days, radiances correspond always to clear sky radiances, but smaller radiances on other days may also correspond to clear sky situations. If the time period is shortened, (5 days), the maximum IR radiance image may remain polluted by some permanent clouds. Consequently, the stability which appears in the VIS and IR surface radiances appears insufficient to allow the use of simple thresholds relative to the minimum visible and/or maximum infrared images for the cloud/surface separation.

In order to determine the radiances representative of the cloud classes for the whole period, VIS-IR bidimensional histograms were built on chosen areas for each day, and then condensed in a single one for the whole period. Characteristic shapes appear, related to high clouds, cirrus, middle-level clouds, low clouds and surface. The distribution of the pixels inside these histograms has been interpreted in terms of variations of cloud cover and optical thickness. However, many pixels from different cloud layers and some from the surface can be mixed in the same area of the histogram when 1) cloud coverage of the pixels is partial, 2) cloud optical thickness is low or 3) when the surface temperature is relatively cold. In these cases, the spectral information appears insufficient to separate cloud types, and some kind of spatial information, related to the cloud cover structure, has to be introduced. The study of local spatial variance images (both in infrared and visible), and analysis of the corresponding histograms showed that a better separation is possible, primarily between homogeneous layers and surface, but also between thin clouds and partial coverage induced by different cloud layers.

Results of the application of a statistical technique accounting both for the radiances and the local variances confirm the usefulness of these parameters for cloud/surface separation, as well as for separation between the cloud types. This is demonstrated by comparisons with results of threshold techniques using the minimum VIS and maximum IR composite images, and results of the same statistical method applied on the radiances only.
A comparison with conventional nephanalyses showed us that the main advantage of the classification is that it gives the characteristic radiances and standard deviations 'corresponding to the classes, which are purely objective parameters-the opposite of cloud names. The classification introduces, then, objective separations between the classes, corresponding to significant changes in the cloud top temperatures, optical thickness and structure. This allows distinction between different kinds of low or medium cloud coverages, and also the separation of cirrus in different classes according to their thickness. This information is supplementary relative to the usual nephanalysis. It is also noted that the classification considers partial coverage of the pixels by cloud as a cloud class, which can produce an overestimation of the cloud cover relative to the one inferred by the nephanalyst, based on larger-scale estimations.

The use of a technique using spectral, spatial and temporal information is shown to be useful in improving the cloud classifications. Time compositing allows reduction of the computation time, as the clustering itself is used only once for a period instead of once for each image. However, the aim of this paper is not to put forward the use of a clustering technique, but to demonstrate the usefulness of simultaneously using spectral and spatial information contained in a time series of images to characterize the different cloud coverages and types. It has been shown that the spatial and temporal scales have to be carefully chosen in order to insure both the stability of the cloud levels and relative stability of the surface properties. Further studies are necessary to assess the influence of these space and time scales; this would be specially useful in the context of ISCCP, where the cloud processing is done on reduced resolution data, as opposed to the present study which is done at full satellite resolution.

In this paper, we have limited our experiments to one particular time of the day, with high solar elevation. The use of visible and visible variances would be precluded for low solar elevations or at night. First experiments on diurnal cycles over West Africa, not described here, show that the use of infrared radiances and infrared variances associated with the water vapor radiances $(5.7-7.1 \mu)$ improves the nighttime classifications and then the continuity between daytime fiveparameters (VIS, IR, VIS Isd, IR lsd, WV) and nighttime three-parameters (IR, IR lsd, WV) classifications.

Acknowledgments. The authors thank the Direction de la Meteorologie Nationale (France) for providing the nephanalysises. They are also grateful to the Goddard Institute for Space Studies where part of this work was done during the stay of one author (Genevieve Seze) in the team of Bill Rossow and to Elaine Matthews for useful discussions and comments about the manuscript. 


\section{REFERENCES}

Arking, A., and J. D. Childs, 1985: Retrieval of cloud cover parameters from multispectral satellite measurements. J. Climate Appl. Meteor., 24, 322-333.

Belcour, C., 1985: Etude de champs de stratocumulus maritimes par l'analyse d'images provenant de satellites geostationnaires. These de Docteur-Ingenieur, Universite Paris VI (Paris) $155 \mathrm{pp}$.

Coakley, J. A., and F. P. Bretherton, 1982: Cloud cover from high resolution scanner data: Detecting and allowing for partially filled fields of view. J. Geophys. Res., 87, 4917-4932.

- and D. G. Baldwin, 1984: Towards the objective analysis of clouds from satellite imagery data. J. Climate Appl. Meteor., 23, 1065-1099.

Desbois, M., and G. Seze, 1984: Use of space and time sampling to produce representative cloud classifications. Ann. Geophys., 2, 599-606.

_- _ and G. Szejwach, 1982: Automatic classification of clouds on Meteosat imagery: application to high level clouds. J. Climate Appl. Meteor., 21, 401-412.

Diday, E., and J. C. Simon, 1980: Clustering analysis. Digital Pattern Recognition, K. S. Fu, Ed. Springer-Verlag, 47-94.

Koepke, P., 1982: Meteosat VIS channel. Signal reduction due to atmospheric water vapor and ozone. Beitr. Phys. Atmos., 55, n 4, pp. 358-369.

Minnis, P., and E. F. Harrison, 1984: Diurnal variability of regional cloud and clear sky radiative parameters derived from GOES data. Part I: analysis method. J. Climate Appl. Meteor., 23, 9931011.

Parikh, J., 1977: A comparative study of cloud classification techniques. Remote Sensing Environ., 6, 67-81.

$\longrightarrow$, and J. T. Ball, 1980: Analysis of cloud type and cloud amount during GATE from SMS infrared data. Remote Sensing Environ., 9, 225-245.

Phulpin, T., M. Derrien and A. Brard, 1983: A two-dimensional histogram procedure to analyse cloud cover from NOAA satellite high resolution data. J. Climate Appl. Meteor., 22, 1332-1345.

Platt, C. M. R., 1981: Two-dimensional histograms of GMS-1 satellite visible albedo and infrared temperature for selected cloud systems. CSIRO Div. Atmos. Phys., Tech. Paper No. 40, 43 pp.

- , 1983: On the bispectral method of cloud parameter determination from satellite VISSR data: separating broken cloud and semi-transparent cloud. J. Climate Appl. Meteor., 22, 429-439.

Rossow, W. B., E. Kinsella, A. Wolf, and L. Garder, 1985: World Climate Research Program. International Satellite Cloud Project (ISCCP) Description of Reduced Resolution Radiance Data, July 1985.

- , F. Mosher, E. Kinsella, A. Arking, M. Desbois, E. Harrison, P. Minnis, E. Ruprecht, G. Seze, C. Simmer and E. Smith, 1985: ISCCP cloud algorithm Intercomparison. J. Climate Appl. Meteor., 24, 877-903.

— L. Garder, E. Kinsella, A. A. Lacis and C. Brest, 1986: Global seasonal cloud variations from satellite radiance measurements. J. Climate Appl. Meteor. (to be published).

Seze, G., F. Drake, M. Desbois and A. Henderson-Sellers, 1986: Total and Low Cloud Amounts over France and Southern Britain in the Summer of 1983: Comparison of Surface-Observed and Satellite-Retrieved Values. Int. J. Remote Sensing 7, m8, 10311050.

Simmer, C., E. Raschke and E. Ruprecht, 1982: A method for determination of cloud properties from two-dimensional histograms. Ann. Meteor., 18, 130-132.

Tsonis, A. A., 1984: On the separability of various classes from GOES visible and infrared data. J. Climate Appl. Meteor., 23, 13931410. 